PROCEEDINGS OF THE

AMERICAN MATHEMATICAL SOCIETY

Volume 130, Number 12, Pages 3571-3578

S 0002-9939(02)06349-9

Article electronically published on July 15, 2002

\title{
SETS THAT FORCE RECURRENCE
}

\author{
ALEXANDER BLOKH AND ADAM FIELDSTEEL
}

(Communicated by Michael Handel)

\begin{abstract}
We characterize those subsets $S$ of the positive integers with the property that, whenever a point $x$ in a dynamical system enters a compact set $K$ along $S, K$ contains a recurrent point. We do the same for uniform recurrence.
\end{abstract}

Let $X$ denote a (compact) metric space and $f: X \rightarrow X$ a continuous map. We will use the term (compact) dynamical system to refer to such a pair $(X, f)$. As usual, we write $f^{n}$ for the $n$-fold composition of $f$ with itself and $\mathbb{N}$ for the set of all positive integers. Let $R(f)$ denote the set of recurrent points of $f$. That is,

$$
R(f)=\left\{x \in X \mid \text { for some sequence of integers } n_{k} \rightarrow \infty, f^{n_{k}}(x) \rightarrow x\right\} .
$$

A well-known theorem (whose origin we have been unable to determine) states the following:

Theorem 1. If $(X, f)$ is a compact dynamical system and $U$ an open set containing $R(f)$, then for all $x \in X$,

$$
\lim _{N \rightarrow \infty} \frac{1}{N} \sum_{n=1}^{N} \chi_{X \backslash U}\left(f^{n}(x)\right)=0 .
$$

We reformulate this theorem as follows. Say that a set $S \subset \mathbb{N}$ has positive upper density if $\lim \sup _{N \rightarrow \infty} \frac{1}{N} \operatorname{card}(S \cap\{1,2, \ldots, N\})>0$.

Theorem 2. If $(X, f)$ is a compact dynamical system and $K \subset X$ is compact, $S \subset \mathbb{N}$ has positive upper density, and for some $x \in X$ and all $n \in S, f^{n}(x) \in K$, then $K \cap R(f) \neq \emptyset$.

This result suggests the following definition.

Definition 1. Say that a set $S \subset \mathbb{N}$ forces recurrence if whenever $(X, f)$ is a (not necessarily compact) dynamical system and $K \subset X$ is compact, and for some $x \in X$ and all $n \in S, f^{n}(x) \in K$, we have $K \cap R(f) \neq \emptyset$.

Thus Theorem 2 asserts that (for compact dynamical systems) every set of positive upper density forces recurrence. The usual proof of the theorem is accomplished by introducing an invariant probability measure for $f$ and invoking the Poincaré recurrence theorem. A closer examination of that proof, however, leads to a somewhat stronger result, and a characterization of all sets that force recurrence. We

Received by the editors November 30, 2000.

2000 Mathematics Subject Classification. Primary 37B20.

The first author was partially supported by NSF grant DMS-9970363.

(C)2002 American Mathematical Society 
begin with a general theorem that allows us to conclude that each member of a suitable class of sets forces recurrence.

If $S \subset \mathbb{N}$ and $m \in \mathbb{N}$, we write $S-m$ to denote $\{s-m \mid s \in S, s-m \geq 1\}$.

Theorem 3. Let $\mathcal{P}$ be a non-empty family of non-empty subsets of $\mathbb{N}$ such that:

1. $(\forall S \in \mathcal{P})(\exists m \in \mathbb{N})$ such that $(S-m) \cap S \in \mathcal{P}$, and

2. $\mathcal{P}$ has the Ramsey property: if $S \in \mathcal{P}$ and $S=\bigcup_{i=1}^{r} C_{i}$, then for some $i \in\{1,2, \ldots, r\}, C_{i} \in \mathcal{P}$.

Then $(\forall S \in \mathcal{P}) S$ forces recurrence.

We note in passing that for such a set $\mathcal{P}$, and all $S \in \mathcal{P}, S$ must be infinite. Indeed, if $S$ were finite, say $S=\left\{s_{1}, \ldots, s_{k}\right\}$, then for some $i,\left\{s_{i}\right\} \in \mathcal{P}$, by the Ramsey property. But a singleton cannot satisfy the first property, since $\emptyset \notin \mathcal{P}$.

Proof. Let $\mathcal{P}$ be a family of subsets of $\mathbb{N}$ as above, and suppose $(X, f)$ is a dynamical system, $K_{1} \subset X$ is compact, $S_{1} \in \mathcal{P}$, and $x \in X$ is a point such that for all $n \in S_{1}, f^{n}(x) \in K_{1}$.

We will show that $K_{1} \cap R(f) \neq \emptyset$.

This will follow when we construct a decreasing sequence of non-empty compact sets $K_{1} \supset K_{2} \supset \ldots$ and a sequence $\left\{m_{i}\right\}_{i=1}^{\infty}$ of positive integers such that for each $i$, $\operatorname{diam}\left(K_{i}\right)<\frac{1}{i}$, and $f^{m_{i}}\left(K_{i+1}\right) \subset K_{i}$. Indeed, these conditions imply that $\bigcap_{i=1}^{\infty} K_{i}$ contains a single point $y$, and that for all $j<k \in \mathbb{N}$,

$$
f^{m_{j}+m_{j+1}+\ldots+m_{k}}(y) \in K_{j} .
$$

Therefore $y$ is recurrent.

Choose $m_{1} \in \mathbb{N}$ so that $\left(S_{1}-m_{1}\right) \cap S_{1} \in \mathcal{P}$. For every $n \in\left(S_{1}-m_{1}\right) \cap S_{1}$ the point $f^{n}(x)$ and the point $f^{n+m_{1}}(x)$ belong to $K_{1}$. It follows that $K_{1} \cap f^{-m_{1}} K_{1}$ is a non-empty compact subset of $K_{1}$. Write

$$
K_{1} \cap f^{-m_{1}} K_{1}=\bigcup_{i=1}^{r_{1}} K_{1, i}
$$

where each $K_{1, i}$ is compact and has diameter less than $\frac{1}{2}$. Let

$$
C_{1, i}=\left\{n \in\left(S_{1}-m_{1}\right) \cap S_{1} \mid f^{n}(x) \in K_{1, i}\right\},
$$

so that $\left(S_{1}-m_{1}\right) \cap S_{1}=\bigcup_{i=1}^{r_{i}} C_{1, i}$. Choose $i_{1}$ so that $C_{1, i_{1}} \in \mathcal{P}$, and set

$$
S_{2}=C_{1, i_{1}} \text { and } K_{2}=K_{1, i_{1}}
$$

Clearly $K_{2} \subset K_{1}, \operatorname{diam}\left(K_{2}\right)<\frac{1}{2}$ and $f^{m_{1}}\left(K_{2}\right) \subset K_{1}$.

We continue inductively: after constructing $S_{i}, K_{i}$ and $m_{i}$ we apply the above argument to $S_{i}$ and $K_{i}$, the only difference being that we cover the corresponding set $K_{i} \cap f^{-m_{i}} K_{i}$ by compact sets of diameter less than $\frac{1}{i+1}$.

Theorem 3 now follows.

Observe that we prove a bit more than claimed. Namely, for the point $y$ from the proof of Theorem [3, there exists a sequence of iterates $f^{n_{i}}(y) \in K_{1}$ such that $f^{n_{i}}(y) \rightarrow y$ (the first condition is not required in the definition of forcing recurrence).

Corollary 1. If $S \subset \mathbb{N}$ has positive upper density, then $S$ forces recurrence.

Proof. Verify that the class $\mathcal{P}^{+}=\{S \subset \mathbb{N} \mid S$ has positive upper density $\}$ satisfies the two hypotheses of the above theorem. 
Indeed, the same argument yields a slightly stronger result. A set $S \subset \mathbb{N}$ is said to have positive upper Banach density if

$$
\lim _{N-M \rightarrow \infty} \sup \frac{1}{N-M} \operatorname{card}\{S \cap\{M+1, M+2, \ldots, N\}\}>0 .
$$

The class $\mathcal{P}^{b+}$ of sets of positive upper Banach density also satisfies the hypotheses of the theorem, and therefore its elements also force recurrence.

We remark that in verifying the first of the hypotheses, one sees the trace of the Poincaré recurrence theorem which is used in the usual proof.

Now we can characterize those subsets of $\mathbb{N}$ which force recurrence.

Definition 2. Let $\left\{b_{n}\right\}_{n=1}^{\infty}$ be a sequence in $\mathbb{N}$. We let

$$
I P\left\{b_{n}\right\}_{n=1}^{\infty}=\left\{\sum_{k=1}^{r} b_{i_{k}} \mid r \in \mathbb{N}, i_{1}<i_{2}<\ldots<i_{r}\right\} .
$$

Any such set is called an $I P$ set (in $\mathbb{N}$ ). For each $L \in \mathbb{N}$, the initial L-segment of $I P\left\{b_{n}\right\}_{n=1}^{\infty}$ is the set

$$
I P\left\{b_{n}\right\}_{n=1}^{L}=\left\{\sum_{k=1}^{r} b_{i_{k}} \mid r \in \mathbb{N}, i_{1}<i_{2}<\ldots<i_{r} \leq L\right\} .
$$

We say that $S \subset \mathbb{N}$ contains a broken IP set if there is a sequence $\left\{b_{n}\right\}_{n=1}^{\infty}$ in $\mathbb{N}$ such that for each $L \in \mathbb{N}$, there exists $a_{L} \in \mathbb{N}$ with

$$
a_{L}+I P\left\{b_{n}\right\}_{n=1}^{L} \subset S .
$$

We also say in this case that $S$ contains a broken $I P\left\{b_{n}\right\}_{n=1}^{\infty}$.

The terminology here is, of course, derived from Furstenberg and Weiss [2]. Note that we have not formulated a notion of a broken IP set, only that of a set containing a broken IP set. These sets have appeared implicitly in connection with the lemma of Hilbert discussed in [1], p. 26. The proof in [1] shows that for every partition of $\mathbb{N}$, some cell of the partition contains a broken IP set (though the statement of that lemma is slightly weaker).

We will make use of the following theorem of Hindman.

Theorem 4 (Hindman [5]). If $S \subset \mathbb{N}$ is an IP set, $n \in \mathbb{N}$, and $C: S \rightarrow\{1, \ldots, n\}$, then for some $i \in\{1, \ldots, n\}, C^{-1}\{i\}$ contains an IP set.

Theorem 5. A set $S \subset \mathbb{N}$ forces recurrence if and only if $S$ contains a broken IP set.

Proof. Let $\mathcal{P}^{b I P}=\{S \subset \mathbb{N} \mid S$ contains a broken IP set $\}$. We show that $\mathcal{P}^{b I P}$ satisfies hypotheses 1 and 2 of Theorem 3

Let $S \in \mathcal{P}^{b I P}$ and suppose that $S$ contains a broken $I P\left\{b_{n}\right\}_{n=1}^{\infty}$. First we establish property 1 for $S$. Indeed, we show that for all integers $M>0,\left(S-b_{M}\right) \cap S$ contains a broken $I P\left\{b_{n}\right\}_{n=1, n \neq M}^{\infty}$.

Fix $M \in \mathbb{N}$, and for each $L \in \mathbb{N},(L>M)$, choose $a_{L}$ so that

$$
a_{L}+I P\left\{b_{n}\right\}_{n=1}^{L} \subset S .
$$


Then

$$
\begin{aligned}
\left(S-b_{M}\right) \cap S & \supset\left(a_{L}+I P\left\{b_{n}\right\}_{n=1}^{L}-b_{M}\right) \cap\left(a_{L}+I P\left\{b_{n}\right\}_{n=1}^{L}\right) \\
& =a_{L}+\left[\left(I P\left\{b_{n}\right\}_{n=1}^{L}-b_{M}\right) \cap\left(I P\left\{b_{n}\right\}_{n=1}^{L}\right)\right] \\
& \supset a_{L}+\operatorname{IP}\left\{b_{n}\right\}_{n=1, n \neq M}^{L} .
\end{aligned}
$$

To establish property 2 for $S$, we make a diagonal argument reducing the problem to Hindman's Theorem [5. Suppose that $S=\bigcup_{i=1}^{r} C_{i}$. We may suppose that the sets $C_{i}$ are pairwise disjoint, so that we have a function $C: S \rightarrow\{1,2, \ldots, r\}$ given by $C(n)=i$ if and only if $n \in C_{i}$. For each $L \in \mathbb{N}$ let $a_{L} \in \mathbb{N}$ be chosen so that

$$
a_{L}+I P\left\{b_{n}\right\}_{n=1}^{L} \subset S .
$$

Define $C^{L}: I P\left\{b_{n}\right\}_{n=1}^{\infty} \rightarrow\{1,2, \ldots, r\}$ by setting

$$
C^{L}(k)= \begin{cases}C\left(a_{L}+k\right), & \text { when } k \in I P\left\{b_{n}\right\}_{n=1}^{L}, \\ 1, & \text { otherwise. }\end{cases}
$$

Let $\bar{C}: I P\left\{b_{n}\right\}_{n=1}^{\infty} \rightarrow\{1,2, \ldots, r\}$ be a limit point of the sequence $\left\{C^{L}\right\}_{L=1}^{\infty}$ in $\{1,2, \ldots, r\}^{I P\left\{b_{n}\right\}_{n=1}^{\infty}}$. By Hindman's theorem, for some $i \in\{1,2, \ldots, r\}, \bar{C}^{-1}(i)$ contains an IP set, say $I P\left\{d_{n}\right\}_{n=1}^{\infty}$. Therefore, $C^{-1}(i)$ contains a broken $I P\left\{d_{n}\right\}_{n=1}^{\infty}$. We conclude that every set in $\mathcal{P}^{b I P}$ forces recurrence.

Conversely, suppose that a set $S \subset \mathbb{N}$ forces recurrence. View $S$ as an element of $\{0,1\}^{\mathbb{N}}$ by identifying $S$ with its characteristic function $\chi_{S}$. Let $f:\{0,1\}^{\mathbb{N}} \rightarrow\{0,1\}^{\mathbb{N}}$ be the shift map, and let $X=\overline{\left\{f^{n} \chi_{S}\right\}_{n \in \mathbb{N}}}$ be the orbit closure of $\chi_{S}$ under $f$. These are the points $y \in\{0,1\}^{\mathbb{N}}$ all of whose finite blocks appear in $\chi_{S}$ after translation. That is,

$$
X=\left\{\begin{array}{r}
y \in\{0,1\}^{\mathbb{N}} \mid \\
(\forall m \in \mathbb{N})\left(\exists a_{m} \in \mathbb{N}\right) \text { such that } \\
(\forall n \in\{1,2, \ldots, m\}) \chi_{S}\left(a_{m}+n\right)=y(n)
\end{array}\right\} .
$$

We consider the dynamical system $(X, f)$. Let $K=\{y \in X \mid y(1)=1\}$, which is a (non-empty) compact set. Then $f^{n}\left(\chi_{S}\right) \in K$ whenever $n \in S$, so there exists a point $y \in K \cap R(f)$. But since $K$ is open in $X$ and $y \in R(f)$, we know (cf. Theorem 2.17 of [1]) there is an IP set, say $I P\left\{b_{n}\right\}_{n=1}^{\infty}$, along which $y$ returns to $K$. That is, for all $n \in I P\left\{b_{n}\right\}_{n=1}^{\infty}, y(n)=1$. It follows that $(\forall L \in \mathbb{N})\left(\exists a_{L} \in \mathbb{N}\right)$ such that $\left(\forall n \in I P\left\{b_{n}\right\}_{n=1}^{L}\right) \chi_{S}\left(a_{L}+n\right)=y(n)=1$. So, $S$ contains a broken $I P\left\{b_{n}\right\}_{n=1}^{\infty}$.

Remark 1. We have shown that a sequence forces recurrence if and only if it is a member of a class satisfying the hypotheses of Theorem 3 . We see that the class $\mathcal{P}^{b I P}$ is the largest subset of $2^{\mathbb{N}}$ satisfying the hypotheses of Theorem 3 (largest in the sense of containment).

Remark 2. From Corollary 1 and Theorem 5 we see that every sequence of positive upper density in $\mathbb{N}$ contains a broken IP set. Nevertheless, not every set of positive upper density, or even of positive density, contains a translate of an IP set. This is a result of E. Straus, which can be found in [6]. 
Remark 3. The class $\mathcal{P}^{b I P}$ is an example of a divisible class of subsets of $\mathbb{N}$ in the sense of Glasner [3]. Glasner calls a non-empty set $\mathcal{P} \subset 2^{\mathbb{N}}$ divisible if $(i)$ $\emptyset \notin \mathcal{P},($ ii $) \mathcal{P}$ is hereditary upward: $A \in \mathcal{P}$ and $B \supset A$ implies $B \in \mathcal{P}$, and (iii) $\mathcal{P}$ has the Ramsey property. In fact, given any divisible class $\mathcal{P} \subset 2^{\mathbb{N}}$, if we set

$$
b \mathcal{P}=\left\{\widetilde{S} \subset \mathbb{N} \mid(\exists S \in \mathcal{P})(\forall L \in \mathbb{N})\left(\exists a_{L} \in \mathbb{N}\right) a_{L}+(S \cap[1, L]) \subset \widetilde{S}\right\},
$$

then $b \mathcal{P}$ is again divisible. (Only property $(i i i)$ requires proof, and this is done by the diagonal argument used in the proof of Theorem [5) In particular $\mathcal{P}^{b I P}=b \mathcal{P}^{I P}$, where $\mathcal{P}^{I P}$ is the class of supersets of IP sets. Another example of this construction is seen in $\mathcal{P}^{b+}=b \mathcal{P}^{+}$.

We can extend the above result by considering more general forms of recurrence. Let $\mathcal{P}$ be a non-empty family of subsets of $\mathbb{N}$. It will be natural to assume that $\mathcal{P}$ is hereditary upward: $S \in \mathcal{P}$ and $S \subset S_{1}$ imply $S_{1} \in \mathcal{P}$. Suppose $(X, f)$ is a dynamical system. A point $x \in X$ is called $\mathcal{P}$-recurrent if for every neighborhood $U$ of $x,\left\{n \mid f^{n}(x) \in U\right\} \in \mathcal{P}$. We denote the set of $\mathcal{P}$-recurrent points of $(X, f)$ by $R_{\mathcal{P}}(f)$. Such notions of recurrence can be found in the literature as early as 4]. Analogously to the foregoing, we say that a set $S \subset \mathbb{N}$ forces $\mathcal{P}$-recurrence if, whenever $(X, f)$ is a dynamical system and $K \subset X$ is compact, and for some $x \in X$ and all $n \in S, f^{n}(x) \in K$, we have $K \cap R_{\mathcal{P}}(f) \neq \emptyset$. If $S \in b \mathcal{P}$ we say that $S$ contains a broken $\mathcal{P}$-set. We have the following general result, proved exactly like the corresponding fact for ordinary recurrence:

Theorem 6. Let $\emptyset \neq \mathcal{P} \subset 2^{\mathbb{N}}$. If a set $S \subset \mathbb{N}$ forces $\mathcal{P}$-recurrence, then $S$ contains a broken $\mathcal{P}$-set.

We note that ordinary recurrence can be regarded as $\mathcal{P}$-recurrence, where $\mathcal{P}$ is taken variously to be the family of non-empty sets, the family of infinite sets or the family of sets containing an IP set. Consequently, the above theorem implies the corresponding portion of Theorem 5 .

A converse of Theorem $[6$ is not in general available, since there may be compact dynamical systems with no $\mathcal{P}$-recurrent points, in which case no sequence can force $\mathcal{P}$-recurrence. However, in the following case, we obtain a characterization analogous to Theorem 5 .

Recall that a set $S \subset \mathbb{N}$ is called syndetic if it has bounded gaps:

$$
(\exists g \in \mathbb{N})(\forall m \in \mathbb{N})[m, m+g] \cap S \neq \emptyset .
$$

We refer to such a $g$ as a bound on the gaps of $S$. Let $\mathcal{P}_{s}$ denote the family of syndetic sets in $\mathbb{N}$. Note that the condition of $\mathcal{P}_{s}$-recurrence is what is commonly referred to as uniform recurrence.

Recall also that a dynamical system $(X, f)$ is said to be minimal if there are no non-trivial closed $f$-invariant subsets. A (non-empty) closed invariant set $Y \subset X$ is called minimal if $\left(Y,\left.f\right|_{Y}\right)$ is minimal. We will make use of the following facts. If $x \in X$ is $\mathcal{P}_{s}$-recurrent, then the orbit closure of $x$ is minimal. If $(X, f)$ is minimal and $X$ is compact, then every $x \in X$ is $\mathcal{P}_{s}$-recurrent. Every compact dynamical system contains a minimal set.

Now we can prove the following theorem.

Theorem 7. A set $S \subset \mathbb{N}$ forces $\mathcal{P}_{s}$-recurrence if and only if $S$ contains a broken $\mathcal{P}_{s}$-set. 
Proof. We need only prove the first implication. Suppose that $S$ contains a broken $\mathcal{P}_{s}$-set. That is, for some set $S^{\prime} \in \mathcal{P}_{s}$, and for every $L \in \mathbb{N}$, there exists $a_{L} \in \mathbb{N}$ such that

$$
a_{L}+\left(S^{\prime} \cap[1, L]\right) \subset S .
$$

Let $(X, f)$ be a dynamical system, let $K \subset X$ be compact, and let $x \in X$ be a point such that for all $n \in S, f^{n}(x) \in K$. Let $r$ be the smallest element of $S^{\prime}$. Consider the points $z_{L}=f^{a_{L}+r}(x) \in K$, choose a convergent subsequence $z_{L_{i}}$, and let $z=\lim _{i \rightarrow \infty} z_{L_{i}} \in K$. It follows by continuity that for all $s \in S^{\prime}, f^{s-r}(z) \in K$.

We note that, if $g$ is a bound on the gaps of $S^{\prime}$, then

$$
\overline{\left\{f^{n} z\right\}_{n \in \mathbb{N}}} \subset \bigcup_{i=1}^{g} f^{i} K \text {. }
$$

Indeed, it is immediate that each $f^{n} z \in \bigcup_{i=1}^{g} f^{i} K$, and $\bigcup_{i=1}^{g} f^{i} K$ is closed.

Hence, $\overline{\left\{f^{n} z\right\}_{n \in \mathbb{N}}}$ is a compact invariant set and contains a non-empty minimal set $Y$. Since every $y \in Y$ is then $\mathcal{P}_{s}$-recurrent, it suffices to show that $Y \cap K \neq \emptyset$. Let $y \in Y$. If $y \notin K$, choose $n_{i} \rightarrow \infty$ so that $f^{n_{i}} z \rightarrow y$. For each $i$ there exists $m_{i} \in[1, g]$ such that $f^{n_{i}+m_{i}} z \in K$. Passing to a subsequence, we may assume $m_{i}$ is constantly equal to $m$, and so $f^{n_{i}+m} z \rightarrow f^{m} y \in K$. Since $f^{m} y \in Y$ is also $\mathcal{P}_{s}$-recurrent, we are done.

We can give another description of the property of forcing $\mathcal{P}_{s}$-recurrence, by relating it to minimality.

Definition 3. Say that a set $S \subset \mathbb{N}$ forces minimality if whenever $(X, f)$ is a (not necessarily compact) dynamical system and $K \subset X$ is compact, and for some $x \in X$ and all $n \in S, f^{n}(x) \in K$, there exists a minimal set non-disjoint from $K$.

Proposition 1. A set $S \subset \mathbb{N}$ forces $\mathcal{P}_{s}$-recurrence if and only if $S$ forces minimality.

Proof. Let $S \subset \mathbb{N}$ be a set that forces $\mathcal{P}_{s}$-recurrence. Suppose that $(X, f)$ is a dynamical system, $K \subset X$ is compact, and $x \in X$ is a point such that for all $n \in S$, $f^{n}(x) \in K$. Then there exists a $\mathcal{P}_{s}$-recurrent point $z \in K$ which implies that the orbit closure of $z$ is minimal and intersects $K$ over at least $z$.

Conversely, let $S \subset \mathbb{N}$ be a set that forces minimality. To prove that $S$ forces $\mathcal{P}_{s}$-recurrence, it is sufficient, by Theorem 7 , to prove that $S$ contains a broken $\mathcal{P}_{s^{-}}$ set. To this end we repeat the construction from the last paragraph of the proof of Theorem 5 Using notation from there we have that $f^{n}\left(\chi_{S}\right) \in K$ whenever $n \in S$, so since $S$ forces minimality, there must exist a recurrent point $y \in K$ whose orbit closure is minimal. Therefore the point $y$ enters $K$ along a syndetic set $S^{\prime}$, which, as in the last paragraph of the proof of Theorem 7 , implies that $S$ contains translates of all initial segments of $S^{\prime}$, thus completing the proof.

A further simple variant of these ideas can be found by considering non-wandering behavior. Given a family $\mathcal{P} \subset 2^{\mathbb{N}}$, we say that a point $x$ in a dynamical system $(X, f)$ is $\mathcal{P}$-non-wandering if for every neighborhood $U$ of $x$, we have that $\left\{n \mid f^{n}(U) \cap U \neq \emptyset\right\} \in \mathcal{P}$. We say that a set $S \in 2^{N}$ forces $\mathcal{P}$-non-wandering if whenever a point $x$ in a dynamical system $(X, f)$ enters a compact set $K \subset X$ along a set in $\mathcal{P}$, there must be a $\mathcal{P}$-non-wandering point in $K$. We then have the following proposition. 
Proposition 2. If $\mathcal{P} \subset 2^{\mathbb{N}}$ is a family of non-empty sets which is:

1. shift-invariant: $S \in \mathcal{P}$ implies $S-m \in \mathcal{P}$, for all $m \in \mathbb{N}$,

2. hereditary upward and

3. has the Ramsey property,

then every set $S \in \mathcal{P}$ forces $\mathcal{P}$-non-wandering.

Proof. Let $x$ be a point in a dynamical system $(X, f)$ that enters a compact set $K \subset X$ such that for some set $S \in \mathcal{P}$ and all $n \in S, f^{n}(x) \in K$. Write $K=\bigcup_{i=1}^{r_{1}} K_{i}^{1}$ with compact $K_{i}$ having diameter less than 1 . Choose $S_{1} \subset S, S_{1} \in \mathcal{P}$ such that for some $i_{1} \in\left\{1, \ldots, r_{1}\right\}$, and for all $n \in S_{1}, f^{n}(x) \in K_{i_{1}}^{1}$. Continuing in this manner, we get a decreasing sequence of compact sets $\left\{K_{i_{j}}^{j}\right\}_{j=1}^{\infty}$ with $\lim _{j} \operatorname{diam}\left(K_{i_{j}}^{j}\right)=0$, and a (decreasing) sequence of sets $S_{j} \in \mathcal{P}$ such that for all $n \in S_{j}, f^{n}(x) \in K_{i_{j}}^{j}$. Let $z$ be the unique point in $\bigcap_{j=1}^{\infty} K_{i_{j}}^{j}$. Then if $U$ is a neighborhood of $z$, for some $j, K_{i_{j}}^{j} \subset U$. If $m$ is the first element of $S_{j}$, then we have $f^{m}(x) \in K_{i_{j}}^{j} \subset U$ and $f^{n}\left(f^{m}(x)\right) \in K_{i_{j}}^{j} \subset U$ for all $n \in S_{j}-m \in \mathcal{P}$. Hence $\left\{n \mid f^{n}(U) \cap U \neq \emptyset\right\} \in \mathcal{P}$.

Note that we have proved more than required, namely, for any neighborhood $U$ of $x$, there is a set $S_{U} \in \mathcal{P}$ such that

$$
\bigcap_{n \in S_{U} \cup\{0\}} f^{-n}(U) \neq \emptyset
$$

and not merely that for each $n \in S_{U}$,

$$
f^{-n}(U) \cap U \neq \emptyset .
$$

Two examples of families that have the properties stated in this proposition but whose elements do not force (even ordinary) recurrence are the following:

1. $\mathcal{P}_{1}=\{S \subset \mathbb{N} \mid(\forall k \in \mathbb{N}) S$ contains an arithmetic sequence of length $k\}$ and

2. $\mathcal{P}_{2}=\left\{S \subset \mathbb{N} \mid \sum_{n \in S} \frac{1}{n}=\infty\right\}$.

That $\mathcal{P}_{1}$ satisfies the Ramsey property follows from van der Waerden's theorem 7]. One can readily construct sets in $\mathcal{P}_{1}$ and in $\mathcal{P}_{2}$ which do not contain broken IP sets. To do this, observe that if a set $S=\left\{m_{i}\right\}_{i=1}^{\infty}$ contains a broken $I P\left\{b_{n}\right\}_{n=1}^{\infty}$, then there are infinitely many $i$ such that $m_{i+1}-m_{i}=b_{2}-b_{1}$. However, we can construct a set $S$ which is a disjoint union $S=\bigcup\left\{m_{i}^{j}\right\}_{i=1}^{r_{j}}$ where:

1. $m_{i_{1}}^{j}<m_{i_{2}}^{k}$ for all $j<k$ and all $i_{1}$ and $i_{2}$,

2. each $\left\{m_{i}^{j}\right\}_{i=1}^{r_{j}}$ is an arithmetic sequence with difference $d_{j}$,

3. $\lim _{j \rightarrow \infty} d_{j}=\lim _{j \rightarrow \infty} m_{1}^{j+1}-m_{r_{j}}^{j}=\infty$,

4. for all $j, \sum_{i=1}^{r_{j}} \frac{1}{m_{i}^{j}}>1$.

Such a set $S$ is in both $\mathcal{P}_{1}$ and $\mathcal{P}_{2}$, but each difference arising from elements of $S$ arises only finitely many times, so that it cannot contain a broken IP set.

Remark 4. All the foregoing extends, mutatis mutandis, to continuous actions of $\mathbb{N}^{d}$, for $d>1$. 


\section{REFERENCES}

[1] Furstenberg, H., Recurrence in Ergodic Theory and Combinatorial Number Theory, Princeton University Press, Princeton, NJ, 1981 MR 82j:28010

[2] Furstenberg, H. and Weiss, B., Topological dynamics and combinatorial number theory, J. D'Analyse Math, 34 (1978), pp. 61-85 MR 80g:05009

[3] Glasner, S., Divisible properties and the Stone-Čech compactification, Can. J. Math., 34, No. 4, 1980, pp. 993-1007 MR 82a:54040

[4] Gottschalk, W. H., and Hedlund, G. A., Topological Dynamics, AMS Colloquium Publications, 36, 1955 MR 17:650e

[5] Hindman, N., Finite sums from sequences within cells of a partition of N, J. Combin. Th., A17 (1974), 1-11 MR 50:2067

[6] Hindman, N., Ultrafilters and combinatorial number theory, Number Theory Carbondale 1979, M. Nathanson, ed., Lecture Notes in Math., 751 (1979), 119-184 MR 81m:10019

[7] van der Waerden, B., Beweis einer Baudetschen Vermutung, Nieuw Arch. Wisk. 15 (1927), $212-216$

Department of Mathematics, University of Alabama at Birmingham, UAB Station, Birmingham, Alabama 35294-2060

E-mail address: ablokh@vorteb.math.uab.edu

Department of Mathematics, Wesleyan University, Middletown, Connecticut 06459

E-mail address: afieldsteel@wesleyan.edu 